\title{
Diferencias en la sensibilización a aeroalergenos en pacientes con poliposis nasosinusal
}

\section{Different patterns of sensitization to airborne allergens in patients with nasal polyposis}

Christian Olavarría $L^{1}$, Jessica Salinas $L^{2}$, Michel Royer $F^{1}$, Constanza Valdés $P^{1}$, Consuelo Sanhueza $L^{3}$.

\section{RESUMEN}

Introducción: La poliposis nasosinusal (PN) es una enfermedad inflamatoria crónica de la mucosa. Apoyado en los hallazgos clínicos, histológicos e inmunológicos, se postula a la alergia como un factor etiopatogénico, lo cual no ha sido plenamente demostrado.

Objetivo: Evaluar la presencia de hipersensibilidad a aeroalergenos en pacientes con PN y compararla con pacientes normales.

Material y método: Se realizó prick test a aeroalergenos a todos los pacientes utilizando 30 alergenos frecuentes en el área metropolitana de Santiago, incluyendo pólenes de árboles, malezas y pastos, ácaros, epitelio de animales y hongos habituales. Este test se amplió con el uso de tres hongos habitualmente no evaluados (Stemphyllium, Pullularia, Helminthosporium). Se utilizó la prueba de Chi cuadrado con una significancia de 0,05.

Resultados: $E$ l $71 \%$ de los pacientes con PN $(n=73)$ y el $66 \%$ del grupo control $(n=44)$ presentaban un test cutáneo positivo, sin diferencia estadística significativa. EI patrón de sensibilización a aeroalergenos fue similar, salvo para los alergenos fúngicos: Ios pacientes con PN estaban sensibilizados en $40 \%$ a Pullularia, 30\% a Stemphyllium, y $10 \%$ a Helminthosporium. En tanto que los controles no presentaron sensibilización a estos hongos no habituales.

Discusión y conclusiones: Existe una alta tasa de sensibilización a aeroalergenos en pacientes normales y con PN, lo que sugiere un probable rol de la alergia en la patogénesis de la poliposis nasal. Destaca un patrón diferente de sensibilización a alergenos fúngicos, cuya relevancia clínica debe ser evaluada a futuro.

Palabras clave: Poliposis nasal, hipersensibilidad, hongos.

\begin{abstract}
Introduction: Nasal polyposis (NP) is a chronic inflammatory disease that leads to tissue oedema and eventually polyps. The pathogenesis of NP has not been fully understood yet, but there are clinical, histological, and immunological findings that suggest that allergy plays a role.
\end{abstract}

\footnotetext{
Servicio de Otorrinolaringología, Hospital Clínico de la Universidad de Chile.

Servicio de Inmunología, Hospital Clínico de la Universidad de Chile.

Médico Cirujano Universidad de Chile.
} 
Aim: The aim of this study was to find out the prevalence of airborne allergen hypersensitivity in patients with nasal polyposis.

Material and method: Skin prick test (SPT) was performed to patients with NP and to a control group (CRS). Thirty frequent airborne allergens of the Santiago de Chile metropolitan area, including tree, grass and weed pollens, house dust mites, animal dander and common fungal allergens were tested. This SPT was extended by using three fungal allergens that usually are not evaluated (Stemphyllium, Pullularia, Helminthosporium). Chi square test was used to compare both groups of patients.

Results: $71 \%$ of NP patients ( $n=73$ ) versus $66 \%$ of the controls $(n=44)$ had a positive SPT, although there was no statistical significance. The only difference in sensitization was for fungal allergens. In NP patients, sensitization to Pullularia was about $40 \%, 30 \%$ to Stemphyllium, and 10\% to Helminthosporium. CRS patients were not sensitized to uncommon fungal allergens.

Conclusions: We found a high frequency of sensitization to airborne allergen in CRS and NP patients that suggest a probable role of allergic inflammation in the pathogenesis of nasal polyposis. Stands out a different pattern of sensitization to fungal allergens in the NP group; the clinical relevance of this finding awaits future evaluation.

Key words: Nasal polyps, paranasal sinus diseases, fungal hypersensitivity.

\section{INTRODUCCIÓN}

La poliposis nasosinusal es una condición inflamatoria crónica de la mucosa de la nariz y cavidades perinasales, que afecta entre $1 \%$ y $5 \%$ de la población adulta ${ }^{1,2}$ y representa uno de los tumores benignos más frecuentes en esta localización. A pesar de ser una entidad clínica conocida desde larga data, sus mecanismos etiopatogénicos no han sido completamente dilucidados.

Se reconoce su asociación con patologías inflamatorias crónicas de la mucosa tales como la fibrosis quística, disquinesia ciliar, intolerancia a antiinflamatorios no esteroidales y rinitis eosinofílicas no alérgicas ${ }^{3}$. Con respecto a su patogénesis, la alta frecuencia con que se encuentran eosinófilos y sus mediadores inflamatorios en el tejido polípoideo ha llevado a considerarlos como las células claves en el desarrollo de la poliposis ${ }^{4-6}$. Otras líneas de investigación han demostrado asociación con la sobrexpresión de leucotrienos, la expresión de factores de crecimiento vascular y la formación de anticuerpos Ig $\mathrm{E}$ anti Staphylococcus aureus, como contribuyentes al desarrollo y/o mantención de la poliposis ${ }^{7-9}$.

Durante largo tiempo se planteó la asociación entre poliposis nasosinusal y rinitis alérgica basada en la infiltración eosinofílica y la exacerbación de síntomas en relación a la exposición a alergenos. Sin embargo, estudios epidemiológicos contradicen una relación causal ya que la incidencia de poliposis en atópicos no supera $4 \%$, siendo similar a la de la población general ${ }^{10,11}$. Por el contrario, en otros cuadros nasosinusales asociados a poliposis, como la rinosinusitis fúngica alérgica, se ha logrado demostrar una fuerte relación con la sensibilización a hongos mediada por inmunoglobulina $E$, especialmente de la familia Dermaticiae, mediada por inmunoglobulina $\mathrm{E}^{12-14}$.

Si bien en los últimos años se ha retomado y profundizado el estudio de la hipersensibilidad a distintos alergenos (incluidos hongos y alimentos) en pacientes con poliposis nasosinusal ${ }^{15-17}$ no existen datos clínicos ni epidemiológicos al respecto en nuestro país. Esta situación se ve favorecida por la ausencia habitual de alergenos fúngicos que pudieran ser relevantes en esta patología, en los exámenes alergológicos de rutina.

El objetivo de este trabajo es determinar la asociación entre atopia (demostrable con test cutáneo para aeroalergenos) y poliposis nasosinusal, incluyendo en el estudio familias de hongos no presentes rutinariamente en los exámenes, y comparando los resultados con un grupo de pacientes enrolados consecutivamente, que consultaban al servicio de otorrinolaringología sin patología rinosinusal conocida. 


\section{MATERIAL Y MÉTODO}

\section{Pacientes}

Se reclutaron 73 pacientes adultos en forma consecutiva con evidencias clínicas y endoscópicas de poliposis nasosinusal atendidos en el Servicio de Otorrinolaringología del Hospital Clínico de la Universidad de Chile entre mayo de 2005 a diciembre de 2005. Se excluyeron del estudio aquellos pacientes portadores de alguna enfermedad autoinmune, tratamiento corticoidal sistémico prolongado y mujeres embarazadas. A cada paciente se le realizó una encuesta que consideraba datos epidemiológicos como edad, sexo, antecedentes mórbidos relevantes como asma y atopia, antecedentes quirúrgicos y alergias a drogas.

\section{Controles}

Se reclutaron consecutivamente 44 pacientes adultos durante el mismo lapso de tiempo, sin patología rinosinusal en base a la historia clínica, el examen físico y tomografía computada, sin evidencias endoscópicas de poliposis nasosinusal.

\section{Pruebas cutáneas}

La detección de sensibilización se realizó mediante la prueba de prick test, aplicando una gota de cada uno de 30 extractos alergénicos, de la empresa Jerti (España) excepto en el caso de Pullularia, Stemphyllium y Helminthosporium que pertenecen a la marca Staleyer (Francia) (Tabla 1) en la superficie volar de los antebrazos, puncionando con lancetas de $1 \mathrm{~mm}$ (ALK, Dinamarca), y leyendo el diámetro de pápula a los 15 minutos. Como control positivo se utilizó una solución de histamina ( $10 \mathrm{mg} / \mathrm{mL}$ ) y como control negativo una solución glicerinada.

Se consideró un test positivo a aquel que presentaba un diámetro de pápula mayor 0 igual a 3 milímetros por sobre el control negativo ${ }^{18}$.

\section{Análisis estadístico}

Para la evaluación de los resultados se utilizó la prueba de Chi cuadrado, considerándose significativo un valor de $p<0,05$.

Tabla 1. Extractos alergénicos utilizados

\begin{tabular}{|ll|}
\hline Alergenos (concentraciones) $^{1}$ & \\
\hline Árboles & Otros \\
Acacia $(1 \%)$ & Latex100 HEP/ml \\
Fresno $(1 \%)$ & Intra-domiciliarios \\
Nogal $(1 \%)$ & Dermatofagoides pteronyssinus $(100 \mathrm{HEP} / \mathrm{ml})$ \\
Morera $(1 \%)$ & Dermatofagoides farinae $(100 \mathrm{HEP} / \mathrm{ml})$ \\
Plátano oriental $(100 \mathrm{HEP} / \mathrm{ml})$ & Mezcla de cucarachas $(1 \mathrm{mg} / \mathrm{ml})$ \\
Álamo $(1 \%)$ & Caspa de perro $(30 \mathrm{HEP} / \mathrm{ml})$ \\
Arce $(1 \%)$ & Caspa de gato $(30 \mathrm{HEP} / \mathrm{ml})$ \\
Aromo $(1 \%)$ & Mezcla de plumas $(1 \mathrm{mg} / \mathrm{mL})$ \\
Malezas & Hongos \\
Quenopodio $(100 \mathrm{HEP} / \mathrm{ml})$ & Alternaria $(10 \mathrm{HEP} / \mathrm{ml})$ \\
Plantago $(100 \mathrm{HEP} / \mathrm{ml})$ & Aspergillus $(5 \mathrm{mg} / \mathrm{ml})$ \\
Ambrosia $(100 \mathrm{HEP} / \mathrm{ml})$ & Cladosporium $(5 \mathrm{mg} / \mathrm{ml})$ \\
Pastos & Penicillium $(5 \mathrm{mg} / \mathrm{ml})$ \\
Mezcla de gramíneas $(100 \mathrm{HEP} / \mathrm{ml})$ & Helminthosporium $(1000 \mathrm{IC} / \mathrm{ml})^{\star}$ \\
Festuca $(100 \mathrm{HEP} / \mathrm{ml})$ & Stemphylium $(1000 \mathrm{IC} / \mathrm{ml})^{*}$ \\
Cynodon $(100 \mathrm{HEP} / \mathrm{ml})$ & Pullularia $(1000 \mathrm{IC} / \mathrm{ml})^{*}$ \\
Balllica $(100 \mathrm{HEP} / \mathrm{ml})$ & \\
\hline
\end{tabular}

${ }^{1}$ Todos los extractos alergénicos de Leti España, excepto *, de Stallergenes, Francia. 


\section{RESULTADOS}

En los 73 pacientes con poliposis nasosinusal (PN) la proporción hombres y mujeres fue de 1:2, siendo su promedio de edad 49 años (rango 1882) y en los 44 pacientes del grupo control la razón fue de 1:2 y el promedio 43 años (rango 23-72). No hubo diferencias significativas en la proporción de edad ni sexo de ambos grupos.

El $80 \%$ de los pacientes con PN refería obstrucción nasal como síntoma más relevante y el $23 \%$ tenía antecedente de polipectomía previa. Respecto de los antecedentes mórbidos, 25\% tenía concomitantemente un asma bronquial, y 3 pacientes presentaban intolerancia a la aspirina.

Cincuenta y dos pacientes con poliposis $(71 \%)$ tuvieron al menos una reacción positiva a algún aeroalergeno en el prick test, comparado con 19 pacientes (44\%) del grupo control. El patrón de sensibilización para los aeroalergenos en ambos grupos fue similar, sin diferencias estadísticamente significativas para ninguno de los extractos. Sin embargo se registró una tendencia a una mayor sensibilización a pólenes de árboles en el grupo control, así como un predominio de la sensibilización a hongos, pólenes de pastos, malezas y alergenos intradomiciliarios en los pacientes con PN (Figura 1).
Al analizar separadamente cada una de las especies fúngicas evaluadas se observó que, la sensibilización a hongos no tradicionales (Helminthosporidium, Pullularia y Stemphyllium) sólo se presentó en los pacientes con PN y por el contrario, ningún paciente de este grupo estaba sensibilizado a Aspergillus $(p<0,005)$ (Figura 2).

\section{DISCUSIÓN}

En este trabajo se observa una alta frecuencia de sensibilización a aeroalergenos en el grupo de pacientes con poliposis nasosinusal $(71 \%)$, superior a la prevalencia de atopia esperable en la población general según la literatura $(25 \%)^{19}$. Esta cifra es incluso más alta que los valores encontrados en nuestro grupo control de pacientes y con un estudio previamente realizado en nuestro país en pacientes con sospecha clínica de rinitis alérgica $(63 \%)^{20}$. Nuestros resultados contrastan con los estudios publicados antes del año $2000^{21,22}$, en los que se encuentra una pobre asociación de PN y atopia, pero son concordantes con experiencias más recientes de algunos grupos de investigación ${ }^{15}$. Esta diferencia podría ser explicada por la mejor calidad de los extractos

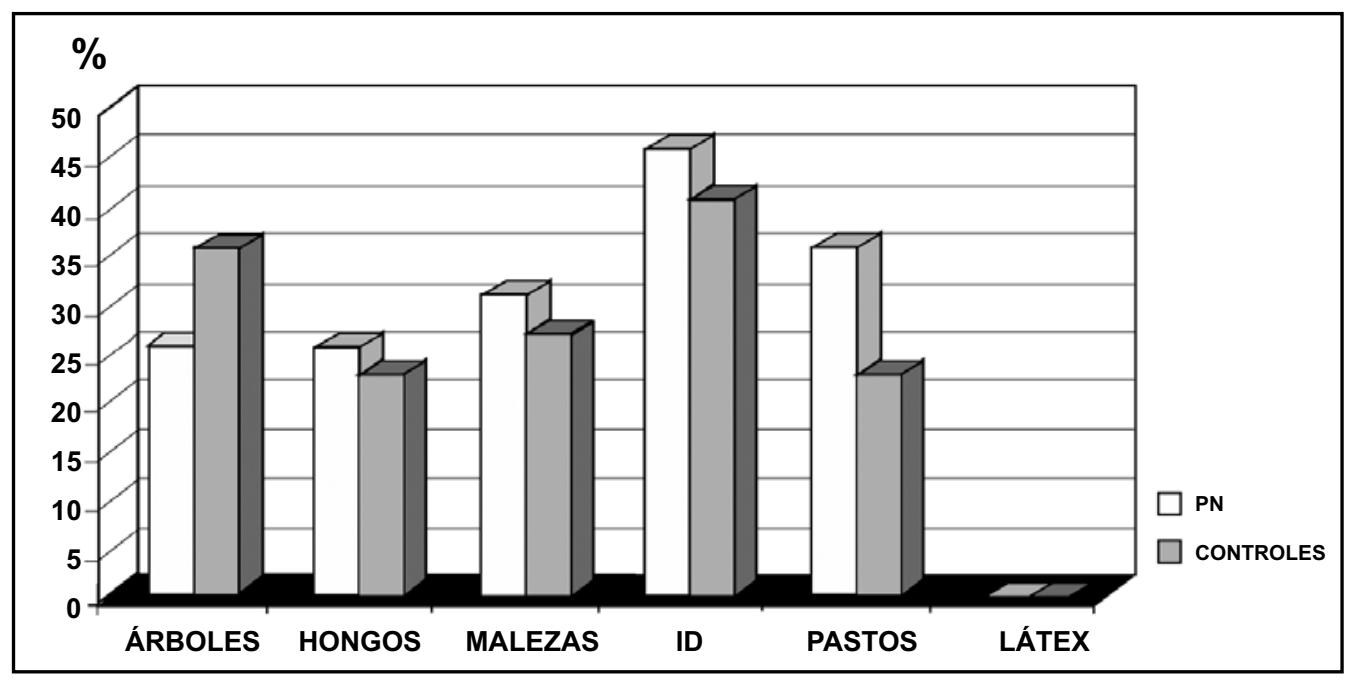

Figura 1. Alergenos en pacientes sensibilizados. ID: Intradomiciliarios (incluye ácaros, cucarachas, caspas de animales y mezcla de plumas), PN: Poliposis Nasosinusal. 


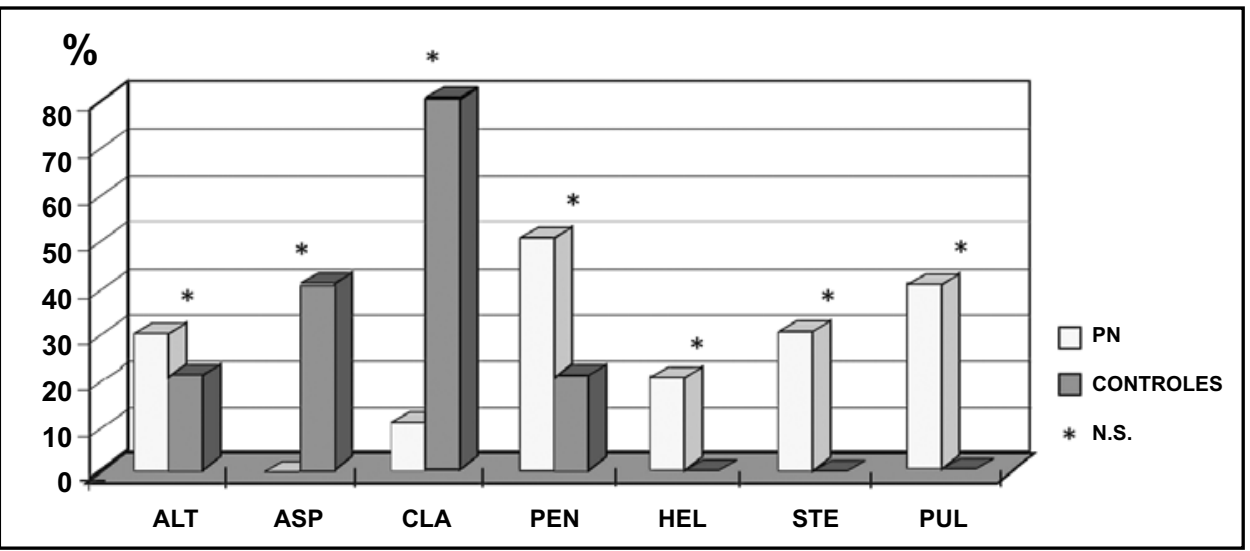

Figura 2. Hongos más frecuentes en pacientes sensibilizados a agentes fúngicos. ALT: Alternaria, ASP: Aspergillus, CLA: Cladosporium, PEN: Penicillium, HEL: Helminthosporium, STE: Stemphylium, PUL: Pullularia. N.S.: No significativo.

alergénicos usados en la actualidad así como por la presencia de sensibilización inhabitual (y tradicionalmente no evaluada en el prick test convencional) en este grupo de pacientes con poliposis nasosinusal, situación que se ve claramente reflejada para el caso de las especies fúngicas. Si bien se observó una tendencia a la sensibilización a alergenos intradomiciliarios en el grupo de pacientes con poliposis $(p<0,05)$, el reclutamiento de un número mayor de pacientes podría confirmar esta diferencia en forma significativa y permitiría sugerir que una exposición permanente a alergenos perennes juega un rol en la aparición y/ o persistencia del fenómeno inflamatorio crónico que acompaña a la formación de los pólipos nasosinusales.

Nuestros pacientes con poliposis nasosinusal presentaron, en un elevado porcentaje, algún grado de sensibilización a aeroalergenos. Su rol en la fisiopatología de esta enfermedad requerirá mayores estudios en este campo, sin embargo queda establecido que aquellos pacientes con poliposis nasosinusal presentan un patrón de sensibilización particular que no estaría siendo estudiado en forma rutinaria en nuestro país, sugiriendo la necesidad de ampliar las baterías de test cutáneo utilizadas actualmemente, al menos, para este grupo de pacientes, considerando que se ha demostrado que el tratamiento concomitante de la rinitis alérgica puede colaborar en el manejo y disminuir la recidiva en pacientes posoperados ${ }^{23}$.

\section{BIBLIOGRAFÍA}

1. Fallers CJ. Familial coincidence of asthma, aspirin intolerance and nasal polyposis. Ann Allergy 1974; 32: 65-9.

2. Hedman J, Kaprio J, Poussa T, Nieminen mM. Prevalence of asthma, aspirin intolerance and chronic obstructive pulmonary disease in a population based study. Int J Epidemiol 1999; 28: 717-22.

3. Bateman ND, Faby C, Woodford TJ. Nasal polyps: still more questions than answers. $J$ Laryngol Otol 2003; 117(1): 1-9.

4. Lamblin C, Gosset P, Salez F, Vandezande LM, Perez T, Darras J et al. Eosinophilic airway inflammation in nasal polyposis. J Allergy Clin Immunol 1999; 14: 85-92.

5. Shin SH, Park JY, Jeon CH, Chol JK, Lee SH. Quantitative analysis of eotaxin and RANTES messenger RNA in nasal polyps: association of tissue and nasal eosinophils. Laryngoscope 2000; 110(8): 1353-7.

6. Woodworth BA, Joseph K, Kaplan AP, Schlosser RJ. Alterations in eotaxin, monocyte chemoattractant protein-4, interleukin-5, and interleukin-13 after systemic steroid treatment for nasal polyps. Otolaryngol Head Neck Surg 2004; 131(5): 585-9.

7. Steinke JW, Bradley D, Arango P, Crouse CD, Frierson H, Kountakis SE, Kraft M, Borish L. Cysteinyl leukotriene expression in chronic 
hyperplastic sinusitis-nasal polyposis: importance to eosinophilia and asthma. J Allergy Clin Immunol 2003; 111(2): 342-9

8. Dagli M, Eryilmaz A, Besler T, Akmansu H, Acar A, Korkmaz $H$. Role of free radicals and antioxidants in nasal polyps. Laryngoscope 2004; 114(7): 1200-3.

9. van Zele T, Gevaert P, Watelet JB, Claeys G, Holtappels G, Claeys C, van Caumenberge P, BACHERT C. Staphylococcus aureus colonization and $\operatorname{lgE}$ antibody formation to enterotoxins is increased in nasal polyposis. J Allergy Clin Immunol 2004; 114(4): 981-3.

10. Settipane GA, Chaffe FH. Nasal polyps in asthma and rhinitis: a review of 6037 patients. J Allergy Clin Immunol 1976; 59: 17-21.

11. Lund VJ. Diagnosis and treatment of nasal polyps. BMJ 1995; 311(7017): 1411-4.

12. Manning SC, Schaefer SD, Close LG, Vuitch F. Culture-positive allergic fungal sinusitis. Arch Otolaryngol 1991; 117: 174-8.

13. Schubert M, Goetz D. Evaluation and treatment of allergic fungal sinusitis. I. Demographics and diagnosis. Journal of Allergy and Clinical Immunology 1998; 102 (3).

14. Mabry RL, Marple B, Mabry C. Mold testing by RAST and skin test methods in patients with allergic fungal sinusitis. Otolaryngol Head Neck Surg 1999; 121: 252-4.

15. KIRTSREESAKUL V. Nasal polyps: the relationship to allergy, sinonasal infection and histopathological type. J Med Assoc Thai 2004; 87(3): 277-82.
16. Dogru $H$, Tuz $M$, Uygur $K$, Akkaya A, Yasan $H$. Asymptomatic lgE mediated food hypersensitivity in patients with nasal polyps. Asian Pac J Allergy Immunol 2003; 21(2): 79-82.

17. PANG YT, EsKICI O, WILSON JA. Nasal polyposis : role of subclinical delayed food hypersensitivity. Otolaryngol Head Neck Surg 2000; 122(2): 298-301.

18. Demoly P, Piette V, Bousquet J. In vivo methods for study of allergy. En: Adkinson NF Jr, Yunginger JW, Busse WW, Bochner BS, Holgate ST, Simons FER, editores. Middleton's Allergy: Principles and Practice. Filadelfia, Estados Unidos: Mosby; 2003; 631-55.

19. Granstrom G, Jacobsson E, Jeppsson PH. Influence of allergy, asthma and hypertension on nasal polyposis. Acta Otolaryngol Suppl 1992; 492: 22-7.

20. FonSECA X. Análisis de resultados de tests cutáneos. Rev Otorrinolaringol Cir Cabeza Cuello 1999; 59: 15-20.

21. Pastorello Ea, Incorvaia C, Riario-Sforza GG, Codecasa L, Menghis V, Bianch C. Importance of allergic etiology in nasal polyposis. Allergy Proc 1994; 15(3): 151-5.

22. Sin A, Terzioglu E, Kokuludag A, Veral A, Sebik F, $\mathrm{K}_{\mathrm{ARCI}} \mathrm{B}, \mathrm{KABAKCI} \mathrm{T}$. Allergy as an etiologic factor in nasal polyposis. J Investig Allergol Clin Immunol 1997; 7(4): 234-7

23. BonfiLs P. Evaluation of combined medical and surgical treatment in nasal polyposis. I: functional results. Acta Otolaryngol 2007; 127(4): 436-46. 